\title{
Evaluation of serum anti-Mullerian hormone levels in women with Hashimoto thyroiditis in the reproductive age
}

Ilknur Ozturk Unsal ( $\nabla$ ilknur_dr@yahoo.com )

Diskapi Yildirim Beyazit Egitim ve Arastirma Hastanesi

\section{Sema Hepsen}

Diskapi Yildirim Beyazit Egitim ve Arastirma Hastanesi

Pinar Akhanli

Diskapi Yildirim Beyazit Egitim ve Arastirma Hastanesi

Murat Calapkulu

Diskapi Yildirim Beyazit Egitim ve Arastirma Hastanesi

Muhammed Erkam Sencar

Diskapi Yildirim Beyazit Egitim ve Arastirma Hastanesi

Ali Yalcindag

Diskapi Yildirim Beyazit Egitim ve Arastirma Hastanesi

\section{Erman Cakal}

Diskapi Yildirim Beyazit Egitim ve Arastirma Hastanesi

\section{Research article}

Keywords: Hashimoto thyroiditis, anti-Mullerian hormone, ovarian reserve, miscariage

Posted Date: November 5th, 2020

DOI: https://doi.org/10.21203/rs.3.rs-39440/v2

License: (9) This work is licensed under a Creative Commons Attribution 4.0 International License. Read Full License 


\section{Abstract}

Background: Autoimmune thyroid disease in women is associated with subfertility and early pregnancy loss, and patients with primary ovarian insufficiency have a high prevalence of thyroid autoimmune disorders. The aim of this study was to investigate the association between Hashimoto thyroiditis $(\mathrm{HT})$ and ovarian reserve.

Methods: Levels of serum thyroid stimulating hormones, thyroid autoantibodies, and anti-Müllerian hormone (AMH) were measured in women with HT and a healthy control group between 2018 and 2019.

Result: Evaluation was made of 108 premenopausal women with HT. A control group was formed of 172 healthy female subjects with normal anti-thyroid antibody levels and thyroid function. Serum AMH levels were determined to be significantly lower in the HT group compared to the control group.

Conclusion: Ovarian reserve evaluated by serum AMH concentration is affected by thyroid autoimmunity independently of anti-thyroid antibodies type or titers.

\section{Background}

In women of reproductive age, thyroid dysfunction is the most common endocrine disorder and the most prevalent cause is thyroid autoimmunity [1]. The thyroid gland, located on the apical membrane of thyrocytes, expresses thyroid peroxidase(TPO), which binds to active iodine and links to iodinated tyrosine residues to form thyroid hormones. Thyroid peroxidase antibodies (TPOAb) are present in almost $90 \%$ of Hashimoto thyroiditis $(\mathrm{HT})$ cases. In reproductive-age women, the prevalence of TPOAb is $8-14 \%$ $[2,5]$. Antithyroglobuline antibodies (TgAb) are specific for thyroglobuline, a matrix protein that contributes to the production of thyroid hormone, and are found in $70 \%$ of patients with HT. Thyroid autoimmune disease prevalence varies between $5 \%$ and $15 \%$ in women of reproductive age [3]. It is not fully known how abnormal thyroid function affects female fertility, but it has been reported that overt and subclinical hypothyroidism may cause menstrual irregularities and anovulation, suggesting that follicular growth and maturation may be impaired by thyroid dysfunction. The reproductive system is directly affected by thyroid dysfunction as oocytes are affected through thyroid hormone receptors on the surface of these cells [4], and there is an indirect effect through the increased prolactin secretion and disrupted $\mathrm{GnRH}$ function $[5,6]$. It has also been reported that thyroid autoimmune disease may be associated with general autoimmunity, resulting in premature ovarian failure. Nevertheless, there has still not been full clarification of the main pathophysiology linking ovarian reserves with TPOAb and thyroid hormones $[6,7]$.

The complex clinical phenomenon of ovarian reserve is influenced by age, genetics, and environmental variables. Biochemical basal and provocative tests and ultrasound imaging of the ovaries are used in ovarian reserve tests. In current clinical practice, the markers most often used are basal folliclestimulating hormone (FSH) levels, antral follicle count, and anti-Müllerian hormone (AMH) [8]. AMH, which is a member of the transforming growth factor- $\beta$ superfamily, is produced by the granulosa cells of 
ovarian antral follicles. It indicates both the number of follicles in the follicle pool, and early antral follicles. As age increases, AMH production decreases, so it may be used for evaluation of the ovarian reserve $[1,2,9,10]$. As $\mathrm{AMH}$ is relatively stable throughout the menstrual cycle in normo-ovulatory women there is no advantage to assessment made by measuring the hormone on a specific day of the menstrual cycle. General guidelines have reported the 5-year group age group classifications of the lower limits of age-appropriate serum $A M H$ values as: $0.5 \mathrm{ng} / \mathrm{mL}$ at 45 years, $1 \mathrm{ng} / \mathrm{mL}$ at 40 years, $1.5 \mathrm{ng} / \mathrm{mL}$ at 35 years, $2.5 \mathrm{ng} / \mathrm{mL}$ at 30 years, and $3.0 \mathrm{ng} / \mathrm{mL}$ at 25 years [8].

Previous studies have shown that thyroid autoimmune disorders have been detected in $12-33 \%$ of patients with primary ovarian insufficiency [11] .Therefore, infertile patients with autoimmune thyroid diseases may have decreased ovarian reserves. In this case, if thyroid autoimmunity has aneffect on follicular growth and development, it may also affect AMH concentrations, independent of female age. The aim of this study was to investigate the correlation between thyroid status, thyroid autoantibodies, and $\mathrm{AMH}$ levels in reproductive aged women.

\section{Methods}

\section{Study population}

This retrospective case-control study included a total of 108 patients with HT with regular menstrual cycles that between 25 and 35 days, both those using and not using levothyroxine, and 172 age-matched healthy female volunteers. Patients younger than 18 years or older than 40 years of age, with autoimmune disease history or polycystic ovary syndrome, using any adjuvant treatment such as glucocorticoids or anticoagulants were excluded. The study was conducted in the Endocrinology Department of Diskapi Yildirim Beyazit Training and Research Hospital between January 2018 and December 2019. The protocol was approved by the Ethics Committee of the University of Health Sciences, Diskapi YildirimBeyazit Training and Research Hospital. The study was conducted in accordance with the Declaration of Helsinki and all participants provided written informed consent before the study procedures. Demographic data were collected together with clinical details and laboratory values.

\section{Clinical, biochemical, and hormonal evaluation}

Baseline demographic data, clinical characteristics (pregnancy and menstrual cycle history), duration of hypothyroidism, and medications were recorded for all study subjects. Hormonal assays were measured, including serum AMH, free thyroxine (FT4), thyroid stimulating hormone (TSH), thyroid autoantibodies, follicle stimulating hormone (FSH), luteinizing hormone (LH), estradiol (E2), and prolactin (PRL). Blood samples were taken from all patients in the morning between 8:00AM and 11:00AM after a 10-hr overnight fast. The serum AMH concentrations were determined by electrochemiluminescence immunoassay performed with Elecys (Roche, Basel, Switzerland). Serum FT4, TSH, TPOAb, and TgAb concentrations, FSH, LH, E2 and PRL levels were measured using ACCEESS 2 immunoassay system (Beckman Coulter, CA, USA) (reference range: FT4, 0.82-1.6 ng/dL; TSH, 0.38-5.33 mIU/L; prolactin, 
3.34-26.72 $\mathrm{ng} / \mathrm{mL}$ ). The reference range for TPOAb and TgAb was $0-9$ and $0-4 \mathrm{IU} / \mathrm{mL}$, respectively at our hospital.

\section{Statistical Analysis}

Statistical analyses were performed using SPSS version 21 software (Chicago, IL, USA). The variables were investigated using visual (histograms, probability plots) and analytical methods (KolmogorovSmirnov/Shapiro-Wilk tests) to assess conformity to normal distribution. The Mann-Whitney U test was performed to compare age, abortus, TSH, fT4, TPOAb and TgAb levels and positivity rates, FSH, LH, estradiol, prolactin, and AMH levels. Descriptive analyses were presented using median and interquartile ranges (IQR) of 25th and 75th percentiles for non-normally distributed variables. A p-value $<0.05$ was considered to show a statistically significant difference. An overall $5 \%$ type- 1 error level was used to infer statistical significance. While investigating the associations between AMH level and other variables, correlation coefficients and their significance were calculated using the Spearman test.

\section{Results}

Evaluation was made of 108 patients with HT and 172 control subjects. While the median age was 32 years (IQR; 27.3-38) for patients and 31 years (IQR; 25-37) for the control group $(p=0.209)$. Of the 108 (38.9\%) HT patients, 42 (38\%) were using levothyroxine. The median disease duration of the patients was 4 years (IQR; 1.9-6.3). The majority of the study subjects had a history of at least one pregnancy, and miscarriage was reported in $8(7.4 \%)$ HT patients and $4(2.3 \%)$ of the control group $(p=0.041)$. The median TSH level was $3.1 \mathrm{mIU} / \mathrm{L}(\mathrm{IQR} ; 1.5-4.6)$ in the HT patient group and $2.1 \mathrm{mIU} / \mathrm{L}$ (IQR; 1.4-2.9) in the control group. The TSH levels of the patients were found to be significantly higher than those of the control group $(p=0.001)$. fT4 levels were similar in both groups $(p=0.497)$. The median TSH was determined as $2.8 \mathrm{mIU} / \mathrm{L}$ (IQR 25-75;1.56-4.65)in patients with euthyroid Hashimoto thyroiditis.

The median TPOAb level of the patient group was 102 (94.4\%) IU/mL and TgAb level was 46 (45.1\%) $\mathrm{IU} / \mathrm{mL}$. The TPOAb and TgAb levels of the patient group were significantly higher than those of the control group ( $p<0.001$ for each). The FSH, LH, estradiol, and prolactin levels of the patients were similar to those of the control subjects $(p=0.684, p=0.854, p=0.064, p=0.162$; respectively). The median AMH level was $1.53 \mathrm{ng} / \mathrm{mL}$ (IQR; 0.77-3.6) in the HT group and $2.3 \mathrm{ng} / \mathrm{mL}$ (IQR; 1.1-3.8) in the control group. AMH levels were found to be lower in the HT patients compared to the control group $(p=0.047)$ (Figure 1$)$. AMH levels in $35.2 \%(n=38)$ of HT patients and in $23.8 \%(n=41)$ of the control group were determined to be $<1 \mathrm{ng} / \mathrm{mL}(\mathrm{p}=0.04)$. The baseline data, laboratory parameters, and AMH levels are presented in Table 1. 
Table 1

Baseline data and laboratory parameters belonging to Hashimoto thyroiditis patients and controls

\begin{tabular}{|llll|}
\hline & Hashimoto patients & Controls & P value \\
\hline Number,n & 108 & 172 & \\
\hline Age, years & $32(27.3-38)$ & $31(25-37)$ & 0.209 \\
\hline LT4 usage, n (\%) & $42(38.9)$ & - & - \\
\hline Disease duration, years & $4(1.9-6.3)$ & - & - \\
\hline Patients with abortions, n (\%) & $8(7.4)$ & $4(2.3)$ & 0.041 \\
\hline Abortion number, n (\%) & $1(1-1)$ & $1(1-2)$ & 0.642 \\
\hline TSH (mIU/L) & $3.1(1.5-4.6)$ & $2.1(1.4-2.9)$ & 0.001 \\
\hline fT4(ng/dL) & $0.95(0.83-1.1)$ & $0.93(0.8-1.1)$ & 0.497 \\
\hline TPOAb(IU/mL) & $195(72-402)$ & $0.7(0.4-1.1)$ & $<0.001$ \\
\hline TPOAb positivity, n (\%) & $102(94.4)$ & $0(0)$ & - \\
\hline TgAb(IU/mL) & $92.2(0.9-158)$ & $0.9(0.9-0.9)$ & $<0.001$ \\
\hline TgAb, positivity, n (\%) & $46(45.1)$ & $0(0)$ & - \\
\hline FSH (IU/L) & $4.3(6.1-8.1)$ & $6(4.1-8)$ & 0.684 \\
\hline LH (IU/L) & $5.3(3.2-7.4)$ & $4.8(3.3-7.3)$ & 0.854 \\
\hline Estradiol (ng/L) & $75(45-106)$ & $54(36-99.9)$ & 0.064 \\
\hline Prolactin (ng/mL) & $11.6(8.6-14.5)$ & $12(9-16.6)$ & 0.162 \\
\hline AMH (ng/mL) & $1.53(0.77-3.6)$ & $2.3(1.1-3.8)$ & 0.047 \\
\hline AMH < 1 ng/mL subjects, n (\%) & $38(35.2)$ & $41(23.8)$ & 0.04 \\
\hline $\begin{array}{l}\text { Categorical data demonstrated with numbers and percentages } \\
\text { presented as medians (interquartile ranges } 25-75) .\end{array}$ & \multicolumn{2}{c|}{ Other variables were } \\
\hline
\end{tabular}

The AMH level was found to be negatively correlated with age $(r=-0.489 p=<0.001)$. There was no association between AMH level and TSH, fT4, TPOAb and TgAb levels, and disease duration (Table 2).

\begin{tabular}{|lll|}
\hline \multicolumn{3}{|c|}{ Table 2 } \\
\multicolumn{3}{|c|}{$\begin{array}{c}\text { Correlation analysis results of AMH } \\
\text { levels and other parameters }\end{array}$} \\
\hline \multicolumn{1}{|c|}{$\mathbf{R}$} & $\boldsymbol{p}$ value \\
\hline Age & -0.489 & $<\mathbf{0 . 0 0 1}$ \\
\hline Disease duration & -0.055 & 0.729 \\
TSHlevel & 0.023 & 0.700 \\
fT4level & 0.016 & 0.796 \\
TPOAb level & -0.084 & 0.173 \\
\hline TgAb level & -0.047 & 0.471 \\
\hline
\end{tabular}


No significant difference in AMH levels was determined between subjects who had or had not had a miscarriage and those who were using or not using levothyroxine $(p=0.366, p=0.734$; respectively). No correlation was determined between autoantibody count (TPO antibody, Tg antibody and both TPO and $\mathrm{Tg}$ antibody-positive) and AMH levels $(\mathrm{p}=0.543)$. No significant difference was determined in AMH levels between HT patients who were positive for TPOAb or $\operatorname{TgAb}(p=0.163, p=0.992$; respectively). The number of patients with AMH level $<1 \mathrm{ng} / \mathrm{mL}$ was similar in the subgroups according to TPOAb or TgAb positivity $(p=0.098, p=0.744$; respectively).

\section{Discussion}

Our study results show that women with $\mathrm{HT}$ have a lower AMH levels compared with age-matched healty women. Thyroid hormone disorders are associated with menstrual irregularities, anovulation, disturbed folliculogenesis and infertility. Menstrual regularity and ovarian reserve play a critical role in achieving pregnancy in reproductive-age women. Premature ovarian failure (POF) is gonadal failure defined by clinical and laboratory findings before the age of 40 . AMH is accepted as a reliable marker for the quantitative evaluation of ovarian reserve, and serum $\mathrm{AMH}$ concentration varies with ageing of the female. Ovarian expression of AMH starts in fetal life, reaches a peak at puberty, starts to decline in adulthood and does not occur after menopause. In women with normal menstrual cycles, levels of serum $\mathrm{AMH}$ have been shown to decrease before FSH rises [12].

Cellular immunity and autoimmune process abnormalitiesplay a role in the autoimmune etiology of POF. There has been stated to be a personal or family history of autoimmune diseasein $80 \%$ of females with idiopathic POF, high titers of thyroid autoantibodies in $50 \%$, and anti-ovary antibodies in $20 \%$. HT is the most frequently seen disease accompanying POF in adult women [13-16].

The hypothesis of this study was that thyroid autoimmunity may suppress follicle development and will therefore reduce the ovarian reserve in patients with $\mathrm{HT}$. The results of this clinical trial showed that serum AMH concentration in the HT patients was lower than in the control group. It was assumed that when antithyroid antibodies are present, they may cause antibody-mediated cytotoxicity in the growing ovarian follicle and damage to the maturing oocyte, thereby leading to diminished quality and developmental potential. In the hypothesis of cross-reactivity, it has been suggested that thyroid autoantibodies alter fertility by targeting zonapellucida, human chorionic gonadotropin receptors and other placental antigens [17]. Weghofer et al reported that thyroid autoimmunity was $11.1 \%$ and serum AMH concentration was $1.3+2.0 \mathrm{ng} / \mathrm{mL}$ in 225 women who underwent IVF. In that study, the mean AMH levels were determined to be significantly higher in women with $\mathrm{TSH}<3.0 \mu \mathrm{IU} / \mathrm{mL}$ compared to those with $\mathrm{TSH} \geq 3.0 \mu \mathrm{IU} / \mathrm{mL}(p=0.02)$ [1]. It was concluded that there was robust statistical evidence that thyroid function in the euthyroid range has a more significant role in the effect on the ovarian reserve than thyroid autoimmunity. However, in the current study, median TSH was 2.8 mIU/L (IQR 25-75;1.564.65) in patients with euthyroid HT. In the 2012 American Association of Clinical Endocrinologists (AACE) and America Thyroid Association (ATA) co-sponsored guidelines, it is stated that the upper limit of a third generation TSH should be considered as $4.12 \mathrm{mIU} / \mathrm{L}$ in iodine-sufficient areas. Morover, approximately 20 
$-26 \%$ of the population could be considered hypothyroid if the upper limit of the normal range were decreased to $2.5-3.0 \mathrm{mIU} / \mathrm{L}[18]$.

Kurado et al showed that AMH levels in women with Hashimoto's thyroiditis were improved with LT4 treatment, but there was no correlation between thyroid antibody titers and serum AMH levels during LT4 supplementation[4]. In contrast in the current study, no differences were determined in serum AMH concentrations of HT patients using or not using levothyroxine (euthyroid HT) $(p=0.734)$. In a study of euthyroid HT adolescents, Pirgon et al demonstrated that AMH levels were significantly higher in the HT group than in the control group [17]. In another study, serum AMH levels were determined to be significantly higher in women with HT than in the control group[12]. From this result, it was suggested that there could be a common etiological link in HT and polycystic ovary syndrome (PCOS). Therefore, PCOS patients were not included in the current study.

As expected, there was determined to be a negative correlation between serum AMH levels and age in this study $(r=-0.489 p=<0.001)$. However, there was no significant correlation of AMH concentration with the levels of TgAb or TPOAb and TSH. Similarly, Chen et al. demonstrated relationship between positive TPOAb and idiopathic low ovarian reserve in Chines women, but this influence was not related TPOAb levels [19]. Osuka et al reported that thyroid autoantibodies are not likely to affect ovarian reserve in euthyroid women with a normal range TSH level, but elevated TSH levels may be involved in decreasing serum AMH levels[10]. Saglam et al. reported lower AMH levels in euthyroid women with HT compared to an age- and BMI-matched healthy control group (1.16 \pm 0.17 vs $1.28 \pm 0.25, p=0.001$ [20]. The results of the current study demonstrated that statistically significantly more HT patients had AMH concentration $<1 \mathrm{ng} / \mathrm{mL}$ compared to the control group (35.2\% vs $23.8 ; \mathrm{p}=0.04)$.

Several studies have shown a correlation between subclinical hypothyroidisim and adverse pregnancy outcomes. The results of the meta-analysis showed that the prevalence of miscarriage in patients with subclinical hypothyroidism with autoimmune thyroiditis was significantly higher compared to those with subclinical hypothyroidism only. However, the effect of subclinical hypothyroidism on the risk of miscarriage is unclear. In the current study the miscarriage rate was statistically significantly higher in the patients with $\mathrm{HT}$, compared to the control subjects ( $p=0.041$ ), but there was no difference in AMH levels between the subjects who had had or had not had a miscarriage $(p=0.366)$. This suggests that autoimmunity itself may increase the risk of miscarriage. Glinoer et al. reported that thyroid autoantibodies indicate abnormal immune function, which induces miscarriage by unstable placenta implantation [21]. This was explained with 3 hypotheses;

The first stated that pregnancy loss is not directly linked to the presence of circulating thyroid antibodies, but rather to a more general autoimmune imbalance, which would therefore explain the higher rejection rate of fetal graft. In the second hypothesis, it is assumed that even if there is apparent euthyroidism, the presence of autoimmune thyroid disease could be related tothe decreased thyroid function capacity to adapt sufficiently to the changes of pregnancy with a reduced functional reserve of thyroid gland. The third hypothesis stated that there was a tendency for pregnancy at an older age in thyroid autoantibody 
positive women and older women are more prone to pregnancy loss [22]. However, further studies on this matter may be needed to reveal the mechanisms.

There were some limitations to this study, primarily the retrospective design and that antral follicle count could not be evaluated. Most studies of thyroid autoimmunity and ovarian reserve have been conducted on infertile women. This study can be considered to contribute to the limited available literature on ovarian reserve in patients with autoimmune thyroiditis. This is the first study to have evaluated $\mathrm{AMH}$ concentrations in women with HT who are euthyroid with levothyroxine treatment and in those who do not use levothyroxine. The women included in this study were not patients who had presented because of infertility.

\section{Conclusion}

The results of this study demonstrated that the ovarian reserve evaluated by serum AMH concentration is affected by thyroid autoimmunity independently of anti-thyroid antiboides type or titers. Factors other than TSH may be involved in the decline of serum AMH levels in adult women with autoimmune thyroiditis. There is a clear need for further studies to more comprehenisvely examine ovarian reserve in HT.

\section{Abbreviations}

HT:Hashimoto thyroiditis; AMH: anti-Müllerian hormone; TPO:Thyroid peroxidase;TPOAb: Thyroid peroxidase antibodies;TgAb: Antithyroglobuline antibodies; FT4: Free thyroxine; TSH;Thyroid stimulating hormone; FSH;Follicle stimulating hormone;LH;Luteinizing hormone;E2; Estradiol; PRL: Prolactin; POF:Premature ovarian failure; PCOS: Polycystic ovary syndrome; AACE; American Association of Clinical Endocrinologists; ATA: America Thyroid Association; BMI: Body mass index

\section{Declarations}

\section{Acknowledgements}

None.

\section{Funding}

No funding sources for research.

\section{Availability of data and materials}

The datasets used and/or analysed during the current study available from the corresponding author on reasonable request.

\section{Authors' contributions}


I.O.U, S.H, P.A, M.C, M.E.S participated in data collection, I.O.U, S.H contributed to interpretation of results, data analyzes, A.Y. analyzed biochemical, and hormonal evaluation I.O.U wrote and edited the manuscript, I.O.U, M.C, S.H contributed to the discussion. I.O.U, E.C contributed to study design, reviewed and edited the manuscript.All authors read and approved the final manuscript.

\section{Ethics approval and consent to participate}

This retrospective study was approved by Diskapi Yildirim Beyazit Traning and Research Hospital Ethics Board (No: 10.07.2019-67/08) and written informed consent of participants were obtained before the study.

\section{Consent for publication}

Non applicable

\section{Competing interests}

The authors declare that they have no competing interests.

\section{Author details}

${ }^{1}$ Department of Endocrinology and Metabolism, University of Health Sciences, Diskapi Yildirim Beyazit Training and Research Hospital, Ankara, Turkey

${ }^{2}$ Department of Biochemistry, University of Health Sciences, Diskapi Yildirim Beyazit Training and Research Hospital, Ankara, Turkey

\section{References}

1. Weghofer A, Barad DH, Darmon S, Kushnir VA, Gleicher N. What affects functional ovarian reserve, thyroid function or thyroid autoimmunity? Reprod Biol Endocrinol. 2016;14(1):26.

2. Vissenberg R, Manders VD, Mastenbroek S, Fliers E, Afınk GB, Ris-Stalpers C.et al. Pathophysiological aspects of thyroid hormone disorders/thyroid peroxidase autoantibodies and reproduction. Hum Reprod Update. 2015;21(3):378-87.

3. Aljarad M, Alhalabi N, Hamad A, Nmr N, Abbas F, Alkhatib A. et al. Prevalence of Thyroid Autoimmune Antibodies in Women Seeking Fertility Care in Damascus, Syria. Cureus. 2019;11(8):e5315.

4. Kuroda K, Uchida T, Nagai S, Ozaki R, Yamaguchi T, Sato Y. et al. Elevated serum thyroid-stimulating hormone is associated with decreased anti-Müllerian hormone in infertile women of reproductive age. J Assist Reprod Genet. 2015;32(2):243-47.

5. Krassas GE, Poppe K, Glinoer D. Thyroid function and human reproductive health. Endocr Rev. 2010;31(5):702-55. 
6. Bahri S, Tehrani FR, Amouzgar A, Rahmati M, Tohidi M, Vasheghani M. et al. Overtime trend of thyroid hormones and thyroid autoimmunity and ovarian reserve: a longitudinal population study with a 12-year follow up. BMC Endocr Disord. 2019;19(1):47.

7. Vadasz Z, Haj T, Kessel A, Toubi E. Age-related autoimmunity. BMC Med. 2013;11:94.

8. Tal R, Seifer DB. Ovarian reserve testing: a user's guide. Am J Obstet Gynecol. 2017;217(2):129-40.

9. Broer SL, Broekmans FJM, Laven JSE, Fauser BCJM. Anti-Müllerian hormone: ovarian reserve testing and its potential clinical implications. Hum Reprod Update. 2014;20(5):688-701.

10. Erol O, Parlak M, Ellidağ HY, Parlak AE, Derbent AU, Eren E. et al. Serum anti-Müllerian hormone levels in euthyroid adolescent girls with Hashimoto's thyroiditis: relationship to antioxidant status. Eur J Obstet Gynecol Reprod Biol. 2016;203:204-9.

11. Osuka S, Iwase A, Goto M, Takikawa S, Nakamura T, Murase T. et al. Thyroid Autoantibodies do not Impair the Ovarian Reserve in Euthyroid Infertile Women: A Cross-Sectional Study. Horm Metab Res. 2018;50(7):537-42.

12. Tuten A, Hatipoglu E, Oncul M, Imamoglu M, Acikgoz AS, Yilmaz N. et al. Evaluation of ovarian reserve in Hashimoto's thyroiditis. Gynecol Endocrinol.2014;30(10):708-11.

13. Özalp Akın E, Aycan Z. Evaluation of the Ovarian Reserve in Adolescents with Hashimoto's Thyroiditis Using Serum Anti-Müllerian Hormone Levels. J Clin Res Pediatr Endocrinol. 2018;10(4):331-35.

14. Košir Pogačnik R, Meden Vrtovec H, Vizjak A, Uršula Levičnik A, Slabe N, Ihan A. Possible role of autoimmunity in patients with premature ovarian insufficiency. Int J Fertil Steril. 2014;7(4):281-90.

15. Dragojević-Dikić S, Marisavljević D, Mitrović A, Dikić S, Jovanović T, Janković-Raznatović S. An immunological insight into premature ovarian failure (POF). Autoimmun Rev. 2010;9(11):771-74.

16. Ayesha null, Jha V, Goswami D. Premature Ovarian Failure: An Association with Autoimmune Diseases. J Clin Diagn Res. 2016;10(10):QC10-2.

17. Pirgon O, Sivrice C, Demirtas H, Dundar B. Assessment of ovarian reserve in euthyroid adolescents with Hashimoto thyroiditis. Gynecol Endocrinol. 2016;32(4):306-10.

18. Biondi B. The normal TSH reference range: what has changed in the last decade? J Clin Endocrinol Metab. 2013;98(9):3584-87.

19. Chen CW, Huang YL, Tzeng CR, Huang RL, Chen CH. Idiopathic low ovarian reserve is associated with more frequent positive thyroid peroxidase antibodies. Thyroid. 2017;27(9):1194-200.

20. Saglam F, Onal ED, Ersoy R, Koca C, Ergin M, Erel O. et al. (2015) Anti-Müllerian hormone as a marker of premature ovarian aging in autoimmune thyroid disease. Gynecol Endocrinol. 31(2):165-68.

21. Zhang $Y$, Wang H, Pan X, Teng W, Shan Z. Patients with subclinical hypothyroidism before 20 weeks of pregnancy have a higher risk of miscarriage: A systematic review and meta-analysis. PLoS ONE. 2017;12(4):e0175708.

22. Glinoer D. Miscarriage in women with positive anti-TPO antibodies:is thyroxine the answer? J Clin Endocrinol Metab. 2006;91(7):2500-02. 
Figures

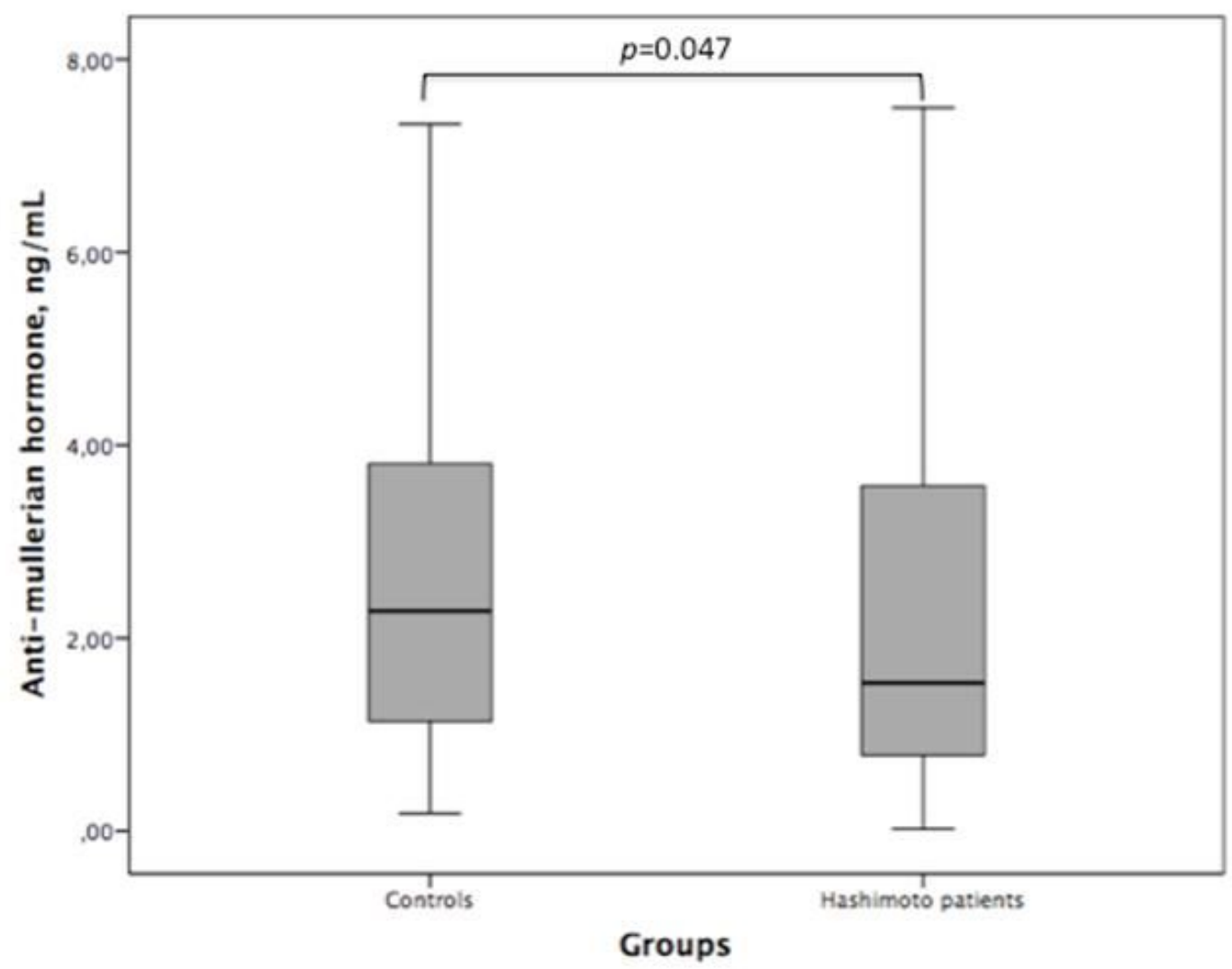

Figure 1. The graphs of anti-mullerian hormone levels of the groups

\section{Figure 1}

The graphs of anti-mullerian hormone levels of the groups 